\title{
Qualidade de vida de mulheres tratadas de câncer de mama
} em uma cidade do nordeste do Brasil

\author{
Quality of life of women treated for breast cancer \\ in a city of the northeast of Brazil
}

Karla Barros Bezerra ${ }^{1}$

Diego Salvador M uniz da Silva ${ }^{2}$

M aria Bethânia da Costa Chein ${ }^{1}$

Patricia Rodrigues Ferreira ${ }^{1}$

Jessika Kelly Passos M aranhão ${ }^{3}$

Nayana Leite Ribeiro ${ }^{3}$

Elba Gomide M ochel $^{1}$

\footnotetext{
${ }^{1}$ Programa de Pósgraduação em Saúde M aterno-Infantil, UniversidadeFederal do M aranhão. Pç. Gonçalves Dias 21/2ㅇ, Centro. 65020-240 São Luís MA. karlabbezerra@yahoo.com.br ${ }^{2}$ FacultadedeM edicina, UniversidadeFederal do Maranhão.

${ }^{3}$ Curso de Fisioterapia, FaculdadeSanta Terezinha.
}

Abstract The study sought to evaluate the quality of life of women treated surgically for breast cancer at the State Oncology Hospital of Reference in São Luís in the State of M aranhão. The quality of life was assessed by the Functional Assessment of Cancer Therapy-Breast (FACT-B). The variables were expressed as mean and standard deviation, and statistical analysis consisted of the AN OVA or Kruskal Wallis test and the Student $t$ or $M$ ann-Whitney test. The level of significance was $\alpha \leq 5 \%$. For statistical tests Stata 9.0 and BioEstat 5.0 were used. 197 women with mean age of $53.0 \pm 11.7$ years participated. The mean values of theFACT-B revealed a good quality of life trend, though the emotional domain was most prejudiced and the cancer sub-scale the most favorable. The type of surgery influenced the social, emotional and cancer sub-scale domain. $N$ on-conservative surgery revealed the worst quality of life levels. A statistically significant relationship between duration of surgery and the physical, emotional and functional domains and between duration of surgery and the scores of the FACT-B was detected. Adjuvant treatment influenced all domains of FACT-B. Q uality of life was relatively good, but was negatively impacted by non-conservative surgery, the shortest time between surgery and the adjuvant therapies. Key words Breast cancer, Quality of life, Mastectomy, FACT-B
Resumo 0 objetivo desteestudo foi avaliar a qualidade devida demulheres tratadas cirurgicamente de câncer de mama no Hospital de Referência Estadual em O ncologia deSão Luís (M A). A qualidadede vida foi avaliada pelo Functional Assessment of Cancer Therapy-Breast (FACT-B). As variáveis foram expressas em média e desvio padrão; e a análise estatística constou da Anova ou Kruskal WalliseTestet deStudent ou M ann-Whitney. 0 nível designificância foi $\alpha \leq 5 \%$. Na estatística foram usados o Stata 9.0 e o BioEstat 5.0. Participaram 197 mulheres com idade média de $53,0 \pm 11,7$ anos. Os valores médios do FACT-B demonstraram tendência para boa qualidade de vida, sendo o domínio Emocional o mais comprometido e a Subescala Câncer a mais favorável. 0 tipo de cirurgia influenciou os domínios Social, Emocional e Subescala câncer do questionário. A cirurgia não conservadora apresentou piores níveis de qualidade de vida. Viu-se relação estatística significante entre o tempo de cirurgia e os domínios Físico, Emocional e Funcional, bem como entre tempo de cirurgia e os escores do FACT-B. 0 tratamento adjuvante influenciou todos os domínios do FACT-B. A qualidade de vida encontrada foi relativamente boa, mas influenciada negativamente pela cirurgia não conservadora, pelo menor tempo desdea cirurgia epelasterapias adjuvantes. Palavras-chave Câncer de M ama, Q ualidade de Vida, M astectomia, FACT-B 
Introdução

O câncer de mama éa neoplasia mais frequente entreas mulheres eo segundo tipo de câncer mais frequenteno mundo. No Brasil, estima-se o surgimento de 52.680 casos de câncer demama, destes, 460 no M aranhão' ${ }^{2}$. Devido à alta incidência, o câncer de mama é provavelmente o mais temido pelas mulheres, sobretudo pelos efeitos advindos do tratamento que podem ser devastadores e mutilantes? ${ }^{2}$.

Alvo de constantes implementos nas últimas décadas, a abordagem terapêutica é composta basicamente pela cirurgia e terapias adjuvantes como a quimioterapia, radioterapia e hormonioterapia. Geralmente, associam-se duas ou mais abordagens, respeitando-se as características clínicas e psicológicas da paciente, para obter uma melhor qualidade de vida (QV) após o tratamento ${ }^{3}$.

A principal abordagem cirúrgica é a mastectomia, queconsistena remoção radical em bloco de todo tecido mamário associado ao esvaziamento axilar, que pode ser completo ou não. Contudo, observa-se que as abordagens mais conservadoras (tumorectomia, quadrantectomia) têm sido preferenciais ${ }^{4} 6$, principalmenteno intuito de reduzir os impactos físicos ${ }^{7}$ e psicológicos ${ }^{8}$ do procedimento cirúrgico.

A mastectomia pode afetar vários segmentos da vida feminina, interferindo inclusive na sua percepção de sexualidade, de imagem corporal e QV 2,9. A depressão, o medo da recidiva, o desconforto físico, a redução das atividades, o distúrbio do sono e as dificuldades sexuais são os principais efeitos advindos desta terapêutica ${ }^{10}$. Dessa forma, conhecer a QV destas mulheres é essencial. Entretanto, dos diversos estudos que tratam do assunto, a maioria é heterogênea em termos metodológicos e controversos em seus resultados.

Os instrumentos utilizados para avaliação da QV podem ser genéricos ou específicos. Os gené ricos avaliam de forma global aspectos relacionados à QV (físico, social, psicológico, emocional, sexual), enquanto os instrumentos específicos são capazes de avaliar de forma direcionada alguns aspectos particulares da QV. Dentre os questionários específicos, o Functional Assessment of Cancer Therapy-Breast (FACT-B) destaca-se por apresentar comprovada confiabilidade e ser fre quentemente aplicado em investigações acerca da QV relacionada ao câncer de mama ${ }^{11-14}$.

Diante do exposto e considerando a relevância do assunto, este estudo busca avaliar a QV de mulheres tratadas de câncer de mama no M aranhão, estado brasileiro onde ainda se percebe relevante precariedade na assistência à saúde e escassez de estudos científicos. Espera-se que os resultados obtidos ampliem os conhecimentos da comunidade científica esubsidiem ações destinadas a garantir a melhoria da QV da população feminina acometida por esta neoplasia.

\section{Metodologia}

Estudo transversal realizado com 197 mulheres tratadas cirurgicamente para o câncer de mama em Hospital de Referência Estadual em Oncologia localizado no município de São Luís (M aranhão, Brasil). A coleta de dados foi realizada durante os meses de Abril a Novembro de 2011 por uma equipe de pesquisadores previamente treinados, esgotando-se as dúvidas acerca das perguntas e possíveis respostas.

A amostra foi composta por mulheres previamente tratadas cirurgicamente para câncer de mama. Tais pacientes foram abordadas na sala de espera do hospital, enquanto aguardavam consulta de acompanhamento pós-cirúrgico. A equipe de pesquisadores entrevistou as pacientes que estiveram no hospital no período de realização do estudo.

Foram incluídas pacientes submetidas a um único tratamento cirúrgico, conservador ou não, para câncer de mama unilateral. Não foram incluídas pacientes com doença recorrente ou me tastática, bem como aquelas submetidas a tratamento por outro câncer.

Para o cálculo amostral, foi considerada a prevalência de $52,98 \%$ de cirurgias para tratamento de câncer de mama no município de São Luís, no ano de 2010 ( número de cirurgias $=213$ e número de casos de câncer de mama $=413$; prevalência $=213 / 402 \times 100=52,98 \%)^{15}$. Admitindo-se intervalo de confiança de $95 \%$ e erro amostral de 5\%, o tamanho amostral resultou em 197 pacientes.

Foi elaborado um questionário com questões semiestruturadas sobre dados sociodemográficos e clínicos das pacientes. As variáveis estudadas foram idade, escolaridade, etnia autodeclarada, estado civil enúmero de filhos. 0 tipo de cirurgia foi investigado e classificado como conservador (que incluía tumorectomia, quadrantectomia ou segmentectomia) ou não, além da presença de tratamento neoadjuvante e adjuvante (quimioterapia, radiotearapia, hormonioterapia), do intervalo de tempo em anos da ci- 
rurgia até o momento da entrevista e da presença de reconstrução mamária.

Todas as participantes assinaram o Termo de Consentimento LivreeEsclarecido. 0 estudo foi previamente aprovado pelo Comitê de Ética em Pesquisa do Hospital Universitário da Universidade Federal do Maranhão.

\section{Avaliação da qualidade de vida}

A qualidade de vida foi avaliada através do Functional Assessment of Cancer Therapy - Breast (FACT-B), versão 4. Através dos reagrupamentos das subescalas do FACT-B foi possível identificar os escores: FACT-G - Functional Assessment of Cancer Terapy - General e TOI Trial O utcome Index. Ambos fazem parte da coleção de questionários de qualidade de vida em doenças crônicas, FACIT - Functional Assessment of Chronic IIIness Therapy (Figura 1).

O FACT-B éum questionário multidimensional composto por 37 questões divididas em cinco domínios que avaliam dimensões distintas do bem- estar da entrevistada: bem-estar físico (GP), bem-estar social efamiliar (GS), bem-estar emocional (GE), bem-estar funcional (GF) e subescala câncer de mama (B).

O FACT-G é composto por 27 perguntas divididas em quatro domínios principais (GP, GS, GE e GF) semelhantemente ao FACT-B. Difere deste pela ausência da subescala câncer de mama (B) , o que permite a avaliação geral de qualquer tipo de câncer.

O escoreTOI - Trial OutcomeIndex éidentificado através da combinação das subescalas:

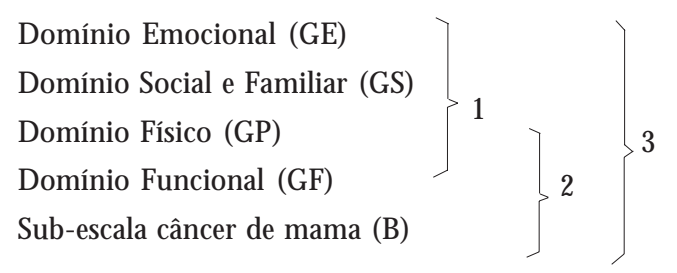
1) FACT-G (Geral)
2) TOI - Trial Outco
3) FACT-B (M ama)

Figura 1. Domínios e subescalas do Functional Assessment of Cancer Therapy - Breast (FACT-B). bem-estar físico (GP), bem-estar funcional (GF) e subescala câncer de mama (B). Composto por 23 itens, o TOI permite explorar a influência do câncer demama nos aspectosfísi cos efuncionais das pacientes.

\section{Análise Estatística}

Os escores do FACT-B, FACT-G e TOI foram calculados e transferidos para planilha eletrônica. Foram demonstradas as médias e desvio padrão (DP) das subescalas do questionário FACT-B, de grupos de classificação com mais de três classes. Aplicou-se os testes de Anova e Kruskal Wallis (teste equivalente, aplicado para variáveis não paramétricas). A comparação das médias das su bescal as nos grupos de apenas duas classes foi realizada a partir do Testet deStudent, para variáveis que seguem a linha de distribuição normal, e do Teste $U$ de M ann-Whitney, para as variáveis não paramétricas. Para digitação e tabulação dos dados, utilizou-se o M icrosoft Office 2007 ®e o pacote estatístico utilizado foi o Stata®(versão 9.0) e o BioEstat ${ }^{\circledR}(5.0)$.

\section{Resultados}

Em relação à caracterização sociodemográfica das pacientes avaliadas, verificou-se idade média de 53,0 anos (DP $\pm 11,7$ ), sendo a faixa etária compreendida entre os 50 e 59 anos a mais prevalente (39\%). A maioria das mulheres era casada (58\%), de cor branca (45\%) e estudaram até o ensino fundamental (46\%) (Tabela 1). Possuíam uma média de 3,2 filhos ( $D P \pm 2,7$ ). A cirurgia não conservadora da mama foi a mais comum (63\%) e foi realizada há menos de 6 anos (93\%). Cerca de $86 \%$ das pacientes não receberam terapias neoadjuvantes e $92 \%$ receberam terapias adjuvantes (Tabela 1 ). O bservou-se ainda que nenhuma paciente foi submetida a reconstrução mamária.

Em relação aos valores médios encontrados para cada domínio do questionário FACT-B, observa-se em todos uma maior aproximação à amplitude máxima possível, tenden do a uma boa qualidade de vida. 0 domínio Emocional foi 0 mais comprometido ea Subescala Câncer a mais favorável. Na avaliação dos escores que associam domínios do questionário, foi encontrada melhor média no FACT-B (Tabela 2).

Ao relacionar variáveis do tratamento para o câncer de mama com os domínios do questionário (Tabela 3), obteve-se associação entre o tipo de cirurgia e os domínios Social $(p=0,023)$, 
Tabela 1. Caracterização amostral das mulheres tratadas do câncer de mama. São Luís, M aranhão, 2012.

\begin{tabular}{|c|c|}
\hline Variáveis & $\begin{array}{c}n=197 \\
n(\%)\end{array}$ \\
\hline \multicolumn{2}{|l|}{ Idade (anos) } \\
\hline 18 a 29 & $3(1)$ \\
\hline 30 a 39 & $19(10)$ \\
\hline 40 a 49 & $45(23)$ \\
\hline 50 a 59 & $77(39)$ \\
\hline 60 a 69 & $34(17)$ \\
\hline Acima de 70 & $19(10)$ \\
\hline M édia \pm DP & $53,0 \pm 11,7$ \\
\hline \multicolumn{2}{|l|}{ Escolaridade } \\
\hline Analfabeta & $11(6)$ \\
\hline Analfabeta funcional & $4(2)$ \\
\hline Ensino Fundamental & $91(46)$ \\
\hline Ensino Médio & $55(28)$ \\
\hline Ensino Superior & $36(18)$ \\
\hline \multicolumn{2}{|l|}{ Etnia autodeclarada } \\
\hline Branca & $88(45)$ \\
\hline Parda & $60(30)$ \\
\hline Negra & $49(25)$ \\
\hline \multicolumn{2}{|l|}{ Estado Civil } \\
\hline Solteira & $45(23)$ \\
\hline Casada & $114(58)$ \\
\hline Divorciada & $10(5)$ \\
\hline Viúva & $28(14)$ \\
\hline \multicolumn{2}{|l|}{ Tipo de Cirurgia } \\
\hline Não conservadora & $125(63)$ \\
\hline Conservadora & $72(37)$ \\
\hline \multicolumn{2}{|l|}{ Tempo de Cirurgia (anos) } \\
\hline$<6$ & $183(93)$ \\
\hline 7 a 12 & $8(4)$ \\
\hline$\geq 12$ & $6(3)$ \\
\hline \multicolumn{2}{|c|}{ Tratamento Adjuvante Précirúrgico } \\
\hline Sim & 27 (14) \\
\hline Não & $170(86)$ \\
\hline \multicolumn{2}{|c|}{ Tratamento Adjuvante Pós-cirúrgico } \\
\hline Sim & $182(92)$ \\
\hline Não & $15(8)$ \\
\hline
\end{tabular}

Emocional $(0,023)$ e Subescala câncer $(p=$ 0,0004). As cirurgias não conservadoras apresentaram os piores níveis de qualidade de vida, exceto no domínio Emocional.

O tempo decorrentedesdea cirurgia influenciou os domínios Físico ( $p=0,001)$, Emocional $(p=0,002)$ efuncional $(p=0,001)$. Embora esta relação não tenha sido significante quando analisada a Subescala Câncer ( $p=0,095)$, nota-se uma tendência para melhora da qualidade de vida com o passar do tempo neste domínio.
Tabela 2. Distribuição das médias, desvio padrão e amplitude dos domínios e escores do questionário FACT-B. São Luís, Maranhão, 2012.

\begin{tabular}{lrcc}
\hline \multicolumn{1}{c}{ Variáveis } & Média & $\begin{array}{c}\text { Desvio } \\
\text { Padrão }\end{array}$ & Amplitude \\
\hline Domínios & & & \\
$\quad$ Físico & 21,1 & 5,4 & $3-28$ \\
Social & 20,8 & 5,6 & $3-28$ \\
Emocional & 17,1 & 3,9 & $8-24$ \\
$\quad$ Funcional & 18,8 & 4,7 & $4-28$ \\
$\quad$ Sub-escala câncer & 24,9 & 5,3 & $9-32$ \\
Escores & & & \\
TOI & 64,7 & 9,8 & $37-84$ \\
FACT - G & 77,8 & 13,2 & $37-102$ \\
FACT - B & 102,6 & 16,7 & $51-131$ \\
& & &
\end{tabular}

${ }^{1}$ Trial OutcomeIndex; ${ }^{2}$ Funcional Assessment of Cancer Treatment-General; ${ }^{3}$ Funcional Assessment of Cancer Treatment-Breast.

A realização de tratamento neoadjuvante e adjuvante (quimioterapia, radioterapia e hormonioterapia) contra o câncer de mama, foi relacionada ao FACT-B. A utilização da terapia após procedimento cirúrgico influenciou de maneira significativa a qualidade de vida relacionada ao câncer de mama ( $p<0,0001)$ (Tabela 3).

Ao relacionar as médias dos escores TOI, FACT-G, E FACT-B com as variáveis do tratamento para o câncer de mama, nota-se influência do tipo $(p=0,036)$ e tempo de cirurgia $(p=$ $0,007)$ sobre as dimensões avaliadas pelo FACTB. Além disso, o tempo desde a cirurgia apresenta associação significante também com aspectos físicos efuncionaisavaliadospelo TOI $(p=0,017)$ e sobre a qualidade de vida geral avaliada pelo escore FACT-G $(p=0,010)$ (Tabela 4).

\section{Discussão}

Os efeitos do tratamento do câncer de mama sobre a QV têm sido amplamente discutidos na literatura-10,12-14,16-30. Uma das formas de avaliálos é através da quantificação da QV em questionários específicos, como o FACT-B 16,17,22,25,31. $N$ este estudo, foram encontrados valores que se aproximaram da amplitude máxima possível para cada D omínio desse questionário, o quetraduz uma QV relativamente boa da população estudada.

Considerando-se os valores encontrados eas amplitudes possíveis para cada Domínio e Escore do questionário, sugere-se avaliar a QV trans- 
Tabela 3. Relação das médias dos domínios do questionário FACT-B com as variáveis referentes ao tratamento para o câncer de mama. São Luís, M aranhão, 2012.

\begin{tabular}{|c|c|c|c|c|c|}
\hline \multirow[b]{2}{*}{ Variáveis } & \multicolumn{5}{|c|}{ Domínios do FACT-B* } \\
\hline & Físico & Social & Emocional & Funcional & Subescala câncer \\
\hline \multicolumn{6}{|l|}{ Tipo de cirurgia } \\
\hline Não conservadora & $20,9 \pm 4,9$ & $20,2 \pm 6,2$ & $17,5 \pm 4,0$ & $18,5 \pm 4,7$ & $23,9 \pm 5,5$ \\
\hline Conservadora & $21,5 \pm 6,3$ & $21,9 \pm 4,3$ & $16,3 \pm 3,5$ & $19,1 \pm 4,7$ & $26,7 \pm 4,4$ \\
\hline p-valor & 0,223 & 0,023 & 0,023 & 0,211 & 0,0004 \\
\hline \multicolumn{6}{|l|}{ Tempo de cirurgia } \\
\hline$<6$ anos & $20,7 \pm 5,4$ & $20,8 \pm 5,7$ & $16,9 \pm 3,9$ & $18,5 \pm 4,7$ & $24,7 \pm 5,4$ \\
\hline 7 a 12 anos & $24,9 \pm 2,5$ & $19,1 \pm 3,1$ & $18,4 \pm 4,4$ & $21,0 \pm 2,3$ & $25,5 \pm 3,8$ \\
\hline$>12$ anos & $26,3 \pm 1,8$ & $19,1 \pm 5,6$ & $22,1 \pm 0,9$ & $24,5 \pm 1,5$ & $29,1 \pm 0,9$ \\
\hline p-valor & 0,001 & 0,552 & 0,002 & 0,001 & 0,095 \\
\hline \multicolumn{6}{|c|}{ Tratamento neo-adjuvante } \\
\hline Sim & $22,0 \pm 4,4$ & $20,0 \pm 4,9$ & $17,8 \pm 4,5$ & $17,9 \pm 4,6$ & $23,4 \pm 6,7$ \\
\hline Não & $21,0 \pm 5,6$ & $20,8 \pm 5,7$ & $17,0 \pm 3,8$ & $18,9 \pm 4,6$ & $25,1 \pm 5,0$ \\
\hline $\mathrm{p}$-valor & 0,197 & 0,237 & 0,179 & 0,147 & 0,069 \\
\hline \multicolumn{6}{|l|}{ Tratamento adjuvante } \\
\hline Sim & $20,9 \pm 5,5$ & $20,3 \pm 5,6$ & $17,2 \pm 3,9$ & $18,5 \pm 4,6$ & $24,4 \pm 1,1$ \\
\hline Não & $23,9 \pm 4,2$ & $25,2 \pm 2,5$ & $15,1 \pm 2,3$ & $20,9 \pm 3,8$ & $29,9 \pm 5,3$ \\
\hline p-valor & 0,018 & 0,0005 & 0,017 & 0,028 & $<0,0001$ \\
\hline
\end{tabular}

${ }^{*}$ Funcional Assessment of Cancer Treatment-Breast.

Tabela 4. Relação das médias dos escores obtidos a partir do questionário FACT-B com as variáveis referentes ao tratamento para o câncer de mama. São Luís, Maranhão, 2012.

\begin{tabular}{|c|c|c|c|}
\hline \multirow[b]{2}{*}{ Variáveis } & \multicolumn{3}{|c|}{ Escores do FACT-B } \\
\hline & TOI* $^{*}$ & FACT-G ${ }^{* *}$ & FACT-B ${ }^{* * *}$ \\
\hline \multicolumn{4}{|l|}{ Tipo de cirurgia } \\
\hline Não conservadora & $63,9 \pm 10,3$ & $77,2 \pm 13,4$ & $101,0 \pm 17,3$ \\
\hline Conservadora & $66,1 \pm 9,0$ & $78,9 \pm 13,3$ & $105,6 \pm 15,9$ \\
\hline p-valor & 0,072 & 0,196 & \\
\hline \multicolumn{4}{|l|}{ Tempo de cirurgia } \\
\hline$<6$ anos & $64,1 \pm 9,9$ & $77,0 \pm 13,3$ & $101,6 \pm 16,8$ \\
\hline 7 a 12 anos & $68,6 \pm 7,0$ & $83,3 \pm 6,8$ & $108,8 \pm 8,7$ \\
\hline$>12$ anos & $74,7 \pm 5,1$ & $92,1 \pm 8,4$ & $121,3 \pm 8,5$ \\
\hline$p$-valor & 0,017 & 0,010 & 0,007 \\
\hline \multicolumn{4}{|l|}{ Tratamento neo-adjuvante } \\
\hline Sim & $62,7 \pm 10,0$ & $77,8 \pm 12,8$ & $101,3 \pm 16,4$ \\
\hline Não & $65,0 \pm 9,8$ & $77,9 \pm 13,3$ & $102,9 \pm 16,8$ \\
\hline p-valor & 0,130 & 0,479 & 0,318 \\
\hline \multicolumn{4}{|l|}{ Tratamento adjuvante } \\
\hline Sim & $62,7 \pm 10,0$ & $77,8 \pm 12,8$ & $101,28 \pm 16,46$ \\
\hline Não & $65,0 \pm 9,8$ & $77,9 \pm 13,3$ & $102,93 \pm 16,86$ \\
\hline $\mathrm{p}$-valor & 0,130 & 0,479 & 0,318 \\
\hline
\end{tabular}

${ }^{1}$ Trial OutcomeIndex; ${ }^{2}$ Funcional Assessment of Cancer Treatment-General; ${ }^{3}$ Funcional Assessment of Cancer Treatment-Breast.

formando-se o valor encontrado em uma percentagem do valor máximo possível para o questionário. Assim, para a amostra estudada, identificaram-seem ordem crescenteas seguintes per- centagens: Domínio Funcional: 67,10\%, Domínio Emocional: $71,33 \%$, Domínio Social: $74,21 \%$, Domínio Físico: 75,32\%, FACT-G: 76,26\%, TOI: 77,05\%, Subescala câncer demama: 78\% eFACT- 
B: $78,35 \%$. Dessa forma, observa-se que todos os escores estão distantes (mais de 20\%) do padrão de QV ideal; sendo o Domínio Funcional o mais comprometido $(67,10 \%)$, e o FACT-B, o menos comprometido (78,35\%).

I nformações acerca da QV da população ge ral seriam esclarecedoras na quantificação e comparação desta variável à população do estudo. A avaliação da QV realizada em estudo comparativo ${ }^{19}$ dos sobreviventes de carcinoma mamário à população geral revelou que sobreviventes apre sentaram escores significativamente inferiores à população geral, exceto nas questões referentes à sexualidade. Houveainda diferenças clínicas importantes nas funções cognitivas, sociais, fadiga, insônia, percepção da imagem corporal, perspectivas futuras, em sintomas relacionados à mama e ao braço ipsilateral da cirurgia e no aspecto financeiro. Por fim, as pessoas tratadas deste câncer apresentavam sintomas mais severos.

A QV tende a sofrer uma maior redução no período pós-operatório, que normalmente está associado a desconforto físico e dor, influenciando principalmente no aspecto funcional do indivíduo. Em estudo ${ }^{16}$ realizado 30 dias após a cirurgia, os valores médios encontrados foram inferiores aos do presente estudo: Domínio Físico $=$ 18,1 $\pm 4,6$; Domínio Social $=18,3 \pm 4,5 ;$ Domínio Emocional $=12,7 \pm 4,2 ;$ Domínio Funcional $=8,4$ \pm 4 , 1; Subescala câncer de mama $=23,0 \pm 4,2$; $\mathrm{TOI}=49,5 \pm 8,8 ; \mathrm{FACT}-\mathrm{B}=80,4 \pm 13,2$. Supõe-se que as mulheres desta amostra passaram mais intensamente por uma série de dificuldades enfrentadas logo após o procedimento cirúrgico, como a incapacidade de trabal har e cuidar de si própria, problemas em satisfazer as necessidades da família, baixa produtividade no trabalho etc., o que justificaria tal achado discordante.

A partir de quatro meses após a cirurgia, as mulheres começam a seadaptar à nova condição (ter câncer/ter feito cirurgia), o que tende a me Ihorar a QV ${ }^{17}$. Talvez por isso tenha sido possível perceber que o tempo de cirurgia influencia a QV das mulheres tratadas, observado de forma bastante evidente nos Domínios Físico, Emocional eFuncional enostrês Escoresalcançadoscom - questionário, além de uma tendência para me Ihora do Domínio Subescala câncer de mama.

Nota-se 0 aumento das médias dos escores FACT-B com o passar do tempo. Mulheres com mais de 5 anos de cirurgia esem recorrência apresentaram melhor QV quando comparadas à pre sente pesquisa ( $F$ ísico $=26,1 \pm 2,87$; Social $=24,0$ $\pm 10,5$; Emocional $=20,0 \pm 3,42$; Funcional $=$ $23,3 \pm 5,06$; Subescala câncer de mama $=24,0 \pm$
4,37; FACT-G $=94,0 \pm 14,0 ;$ FACT-B $=118,2 \pm$ $15,6)^{25}$. Somente na Subescala câncer de mama os achados foram inferiores aos do nosso estudo. Provavelmente, a presença de médias mais baixas nesta pesquisa justificam-se por aspectos metodológicos, uma vez que foram incluídas mulheres com menos de cinco anos de cirurgia.

As cirurgias conservadoras da mama tendem a incrementar a QV no pós-operatório, conforme observado nos Domínios Social e Subescala câncer de mama e no Escore TOI deste estudo. Durante um período de cinco anos após o tratamento cirúrgico, um estudo ${ }^{24}$ comparou a QV de mulheres que fizeram cirurgia conservadora da mama com mulheres tratadas por mastectomia $(n=89)$. No grupo de abordagem conservadora ( $n=226 ; 197$ quadrantectomia ou nodulectomia e 29 reconstrução imediata) também houve me Ihora nos aspectos sociais, assim como neste estudo, além de melhor funcionamento físico edas atividades de escrita, maior atividade sexual e maior satisfação com a imagem corporal desde o primeiro ano após a cirurgia $(p<0,05)$.

Em coorte com 950 mulheres recém-diagnosticadas com câncer de mama, foi verificado que aquelas que não sofreram mastectomia ou nodulectomia tiveram melhores valores nos Domínios Físico $(p=0,0033)$, Funcional $(p=0,0008)$, Subescala câncer de mama ( $p<0,0001)$ e FACT$B(p=0,0018)$ quando comparadas a mulheres mastectomizadas ${ }^{17}$. Novamente, a cirurgia não conservadora aparece como prejudicial à QV.

Especificamente sobre o aspecto físico, sabe se que quanto menos conservadora for a cirurgia, maior a chance do aparecimento de comorbidades. Assim, restrições nos movimentos dos braços tendem a diminuir a QV de mulheres tratadas por cirurgias não conservadoras, comprometendo outros Domínios além do Físico espe cificamente, como o Funcional ea Subescala câncer de mama e, consequentemente, os escores TOI, FACT-G e FACT-B ${ }^{12}$.

Ao avaliar a QV de mulheres atendidas pelo serviço de Fisioterapia, comparando com outras que não passaram pelo serviço de reabilitação física após a cirurgia, observou-se diferença importante entre a avaliação inicial e o pós-tratamento $(p<0,05)$ para os parâmetros limitação física, sexualidade e QV total. Provavelmente como reflexo da melhora da disfunção do ombro e cintura escapular ipsilateral à cirurgia, da reeducação postural e da melhora da percepção da imagem corporal e da autoestima ${ }^{29}$.

Resultados semelhantes foram encontrados em uma coortepopulacional com 1829 mulheres 
chinesas, no qual foi encontrada relação entre atividade física e QV após 6 e 36 meses do diagnóstico decâncer demama. As mulheres que praticavam atividade física no nível metabólico recomendado como ideal apresentaram os escores mais altos para QV total, além dos aspectos físico, psicológico e do Domínio Social, com uma tendência para estabilização dessa relação ao Iongo do tempo ${ }^{18}$.

0 estudo transversal brasileiro com 110 muIheres tratadas a pelo menos 1 ano também demonstrou que as submetidas à mastectomia radical tiveram escores mais baixos queaquelas que fizeram quadrantectomia ou mastectomia radical com reconstrução imediata, ainda que todas apresentassem escores baixos ${ }^{28}$.

As médias de QV (aferidas em três momentos diferentes) de pacientes submetidas à mastectomia com reconstrução imediata foram meIhores que daquelas submetidas à mastectomia sem reconstrução, principalmente nos Domínios Físico, Psicológico, Nível de independência e Relações sociais ${ }^{27}$. Dessa forma, é possível que a QV da mulher mastectomizada no $M$ aranhão seja mais satisfatória ainda, caso a reconstrução imediata da mama seja implementada como terapêutica complementar para a portadora de câncer de mama.

Assim, observando o comportamento da QV frente ao tipo de cirurgia realizada e ao tempo desde a cirurgia, acredita-se quea avaliação clínica e possívei sintervenções em relação à QV imediatamente após o diagnóstico do câncer de mama sejam de suma importância. Seus resulta- dos podem alterar o ajustamento e as funções das mulheres durante a fase de tratamento e mesmo durante o tempo de sobrevivência.

Um segundo aspecto da terapêutica empregada para o câncer de mama que pode influenciar a QV é o tratamento neoadjuvante. Estas terapias não apresentaram relação com a QV, talvez porque tenha sido empregada em um número muito restrito de pacientes. No entanto, a terapia adjuvantecontribuiu para piora da QV nos Domínios Físico, Social, Funcional e Subescala câncer. Na literatura, essa relação ainda se mostra controversa. Há casos em que a quimioterapia está associada a baixos índices de QV nos primeiros 6 meses de tratamento ${ }^{32}$. Enquanto, em pesquisa populacional controlada com 1602 mulheres chinesas, não se evidenciou diferenças na QV entre pacientes que receberam quimioterapia ou radioterapia adjuvantes e aquelas que não receberam ${ }^{21}$.

Finalmente, a QV das mulheres tratadas do câncer de mama no Maranhão parece ter sido negativamente influenciada pela ausência de cirurgia de reconstrução mamária, visto que esta proporciona importante incremento nos escores de QV das mulheres ${ }^{27}$. Tanto aspectos funcionais como psicossociais apresentam relação positiva com a reconstrução mamária pós-mastectomia ${ }^{13,33}$. Porém, a iniciativa para a realização da reconstrução mamária partedo cirurgião na grande maioria dos casos. Vale ressaltar que mesmo em pacientes com nível sociocultural elevado, também há necessidade de orientação profissional para a decisão sobre o tratamento ideal ${ }^{20}$.

\section{Colaboradores}

KB Bezerra trabalhou na concepção, pesquisa e redação. DSM Silva, MBC Chein e EG Mochel trabal haram na metodologia e redação final. PR Ferreira, JKP M aranhão e NL Ribeiro trabalharam na pesquisa. 


\section{Referências}

1. Brasil. M inistério da Saúde (MS). Instituto Nacional de Câncer. Estimativa 2010: incidência de câncer no Brasil: Brasília: MS, INCA; 2009. [acessado 2012 jun 02]. Disponível em: http://www.inca.gov.br/ estimativa/2010/

2. Box RC, Reul-Hirche HM, Bullock-Saxton JE, Furnival CM. Physiotherapy after breast cancer surgery: results of a randomised controlled study to minimise lymphoedema. Breast Cancer Res Treat 2002; 75(1):51-64.

3. National Breast and Ovarian Cancer Centre (NBOCC). Lymphedema: prevalence, risk factors and manage ment. 2009. [acessado 2012 jun 4]. Disponível em: http://canceraustralia.nbocc.org.au/view-documentdetails/lym-lymphoedema- prevalence-risk-factorsand-management-a-review-of-research

4. Veronesi U, Saccozzi R, Del Vecchio M, Banfi A Clemente C, De Lena M, Gallus G, Greco M, Luini A, Marubini E, Muscolino G, Rilke F, Salvadori B, Zecchini A, Zucali R. Comparing radical mastectomy with quadrantectomy, axillary dissection, and radiotherapy in patients with small cancers of the breast. N Engl J Med 1981; 305(1):6-11.

5. Tiezzi DG. Cirurgia conservadora no câncer de mama. Rev Bras GinecolO bstet 2007; 29(8):428-434.

6. Pockaj BA, Degnim AC, Boughey JC, Gray RJ, McLaughlin SA, Dueck AC, Perez EA, Halyard MY Frost MH, Cheville AL, Sloan JA. Quality of life after breast cancer surgery: What have we learned and where should we go next? J Surg Oncol 2009; 99(7):447-455.

7. Cheville AL, Tchou J. Barriers to rehabilitation following surgery for primary breast cancer. J Surg Oncol 2007; 95(5):409-418.

8. Parker PA, Youssef A, Walker S, Basen-Engquist K, Cohen L, Gritz ER, Wei QX, Robb GL. Short-term and long-term psychosocial adjustment and quality of life in women undergoing different surgical procedures for breast cancer. Ann Surg Oncol 2007; 14(11):3078-3089.

9. Rietman JS, Dijkstra PU, Debreczeni R, Geertzen JH, Robinson DP, De Vries J. Impairments, disabilities and health related quality of life after treatment for breast cancer: a follow-up study 2.7 years after surgery. Disabil Rehabil 2004; 26(2):78-84.

10. Lasry JC, Margolese RG, Poisson R, Shibata H, Fleischer D, Lafleur D, Legault S, Taillefer S. Depression and body image following mastectomy and lumpectomy. J Chronic Dis 1987; 40(6):529-534.

11. Brady MJ, Cella DF, Mo F, Bonomi AE, Tulsky DS, Lloyd SR, Deasy S, Cobleigh M, Shiomoto G. Reliability and validity of the Functional Assessment of Cancer Therapy-Breast quality-of-life instrument. J Clin Oncol 1997; 15(3):974-986.
12. Hayes SC, Rye S, Battistutta D, DiSipio T, Newman B. Upper-body morbidity following breast cancer treatment is common, may persist longer-term and adversely influences quality of life. Health Qual Life Outcomes 2010; 8:92.

13. Potter S, Thomson HJ, Greenwood RJ, Hopwood $P$, Winters ZE. Health-related quality of life assessment after breast reconstruction. Br J Surg 2009; 96(6):613-620.

14. Lemieux J, Goodwin PJ, Bordeleau LJ, Lauzier S, Theberge V. Quality-of-life measurement in randomized clinical trials in breast cancer: an updated systematic review (2001-2009). J Natl Cancer Inst 2011; 103(3):178-231.

15. Brasil. Ministério da Saúde (MS). DATASUS - Departamento de Informática do SUS. Informações de Saúde. Brasília: MS, DATASUS; 2010. [acessado 2011 fev 15]. Disponível em: http://www.datasus.gov.br

16. Pandey M, Thomas BC, Ramdas K, Ratheesan K. Early effect of surgery on quality of life in women with operable breast cancer. Jpn J Clin Oncol 2006; 36(7):468-472.

17. Kwan $M L$, Ergas IJ, Somkin CP, Quesenberry $C P J r$. Neugut AI, Hershman DL, M andelblatt J, Pelayo MP, Timperi AW, Miles SQ, Kushi LH. Quality of life among women recently diagnosed with invasive breast cancer: the Pathways Study. Breast Cancer Res Treat 2010; 123(2):507-524.

18. Chen $X$, Zheng $Y$, Zheng W, Gu K, Chen $Z$, Lu W, Shu $X O$. The effect of regular exercise on quality of life among breast cancer survivors. Am J Epidemiol 2009; 170(7):854-862

19. Ahn SH, Park BW, Noh DY, Nam SJ, Lee ES, Lee MK, Kim SH, Lee KM, Park SM, Yun YH. Healthrelated quality of life in disease-free survivors of breast cancer with the general population. Ann Oncol 2007; 18(1):173-182.

20. Elmore L, Myckatyn TM, Gao F, Fisher CS, Atkins J, M artin-Dunlap TM, M argenthaler JA. Reconstruction Patterns in a Single Institution Cohort of Women Undergoing Mastectomy for Breast Cancer. Ann Surg Oncol 2012; 19(10):3223-3229.

21. Cui Y, Shu XO, Gao Y, Cai H, Wen W, Ruan ZX, Jin $F$, Zheng $W$. The long-term impact of medical and socio-demographic factors on the quality of life of breast cancer survivors among Chinese women. Breast Cancer Res Treat 2004; 87(2):135-147.

22. Wenzel LB, Fairclough DL, Brady MJ, Cella D, Garrett KM, Kluhsman BC, Crane LA, M arcus AC. Agerelated differences in the quality of life of breast carcinoma patients after treatment. Cancer 1999; 86(9):1768-1774. 
23. Ishikawa NM. Validação do FACT-F no Brasil e avaliação da fadiga e qualidade de vida em mulheres com câncer de mama [tese]. São Paulo: Universidade Estadual de Campinas; 2009.

24. Arndt V, Stegmaier C, Ziegler H, Brenner H. Quality of life over 5 years in women with breast cancer after breast-conserving therapy versus mastectomy: a population-based study. J Cancer Res Clin Oncol 2008; 134(12):1311-1318.

25. Ohsumi S, Shimozuma K, M orita S, Hara F, Takabatake D, Takashima S, Taira N, Aogi K, Takashima S. Factors associated with health-related quality-of-life in breast cancer survivors: influence of the type of surgery. Jpn J Clin Oncol 2009; 39(8):491-496.

26. Evangelista $A L$, Latorre $M d R D d O$, Ribeiro $K d C B$, Netto M M , Pizão PE. Variação da qualidade de vida em pacientes tratadas com câncer de mama e submetidas a um programa de exercícios aeróbios. RBM Rev Bras M ed 2009; 66(7):200-205.

27. de Oliveira RR, Morais SS, Sarian LO. [Immediate breast reconstruction effects on quality of life of women with mastectomy]. Rev Bras Ginecol Obstet 2010; 32(12):602-608.

28. Huguet PR, M orais SS, Osis MJ, Pinto-N eto AM, Gurgel MS. [Quality of life and sexuality of women treated for breast cancer]. Rev Bras Ginecol Obstet 2009; 31(2):61-67.

29. Moreira ECH, Manaia CAR. Qualidade de vida das pacientes mastectomizadas atendidas pelo serviço de fisioterapia do Hospital Universitário da Universidade Estadual de Londrina. Semina: Ciências Biológicas e da Saúde 2005; 26(1):21-30.

30. Nicolussi AC, Sawada NO. Qualidade de vida de pacientes com câncer de mama em terapia adjuvante. Revista Gaúcha de Enfermagem 2011; 32(4):759766.

31. Gustafson DH, Hawkins R, Pingree S, McTavish F, Arora NK, M endenhall J, et al. Effect of computer support on younger women with breast cancer. J Gen Intern Med 2001; 16(7):435-445.

32. Groenvold M, Fayers PM, Petersen MA, M ouridsen $\mathrm{HT}$. Chemotherapy versus ovarian ablation as adjuvant therapy for breast cancer: impact on health-related quality of life in a randomized trial. Breast Cancer Res Treat 2006; 98(3):275-284.

33. Roth RS, Lowery JC, Davis J, Wilkins EG. Quality of life and affective distress in women seeking immediate versus delayed breast reconstruction after mastectomy for breast cancer. Plast Reconstr Surg 2005; 116(4):993-1002. (Discussion 3-5).

Artigo apresentado em 16/08/2012

Aprovado em 22/11/2012

Versão final apresentada em 30/11/2012 
